\title{
Functional assessment in spinal cord injury: a comparison of the Modified Barthel Index and the 'adapted' Functional Independence Measure
}

Elliot Roth Department of Rehabilitation Medicine, Northwestern University Medical School, Rehabilitation Institute of Chicago Gary Davidoff, John Haughton and Mary Ardner Department of Physical Medicine and Rehabilitation, University of Michigan Medical Centre, Ann Arbor, Michigan

The Modified Barthel Index (MBI) and the Functional Independence Measure (FIM) have been used to provide objective measures of functional status and change of spinal cord injured ( $\mathrm{SCl}$ ) patients. To compare rating scores on the $\mathrm{MBI}$ and $\mathrm{F} \mid \mathrm{M}$, the functional abilities of $41 \mathrm{SCl}$ patients were rated by one trained nurse-clinician using both scales at admission to initial rehabilitation (ADM), discharge from rehabilitation (DC) and at follow-up (FU) 12 months after rehabilitation. An 'adapted' FIM score was used, and total $\mathrm{MBI}$ and FIM scores were divided into self-care and mobility subscores. Comparisons were made between each MBI score and each FIM score at each point in time (ADM, DC, FU) using simple linear regression, which was also used to compare changes in the MBI and FIM scores from ADM to DC and from DC to FU. Excellent correlations $(p<0.0005)$ were found between MBI and FIM scores at all points in time and between changes in $\mathrm{MBI}$ scores and changes in FIM scores over each time interval.

\section{Introduction}

The measurement of functional independence in patients with disabilities has a variety of applications both in patient care and clinical research. The purposes of such an assessment are to provide objective and quantitative measures of patient function; to describe and communicate

Address for correspondence: EJ Roth, Rehabilitation Institute of Chicago, 345 East Superior St, Chicago, Illinois 60611, USA. levels of ability in self-care and mobility skills; to monitor changes in clinical status; to guide management decisions; to evaluate treatment efficacy; to prevent additional disability; to predict prognosis; to plan placement; to estimate care requirements; and to determine compensation. ${ }^{1-4}$ Determination of the type and degree of functional disability is widely accepted as an essential component of the comprehensive rehabilitation evaluation and management of patients with physically disabling conditions. . $^{5-7}$ Recently, the American College of Physicians 
recommended routine application of functional assessment ratings for general medical practice, especially for care of the elderly. ${ }^{2}$ Such evaluations have been shown to result in a number of benefits, including improved identification of previously unrecognized conditions; enhanced diagnostic and therapeutic outcomes; more accurate prediction of clinical complications or problems; more desirable placement of patients; fewer nursing home discharges; reduced durations of hospitalization; earlier recognition of medication overuse; earlier identification of dementia; and improved patient education. ${ }^{8-11}$

Numerous methods may be employed to determine the ability of an individual to perform functional skills. Most evaluations rely on the use of a standardized functional assessment rating instrument. ${ }^{4.12-17}$ Many scales have been developed and utilized, and each rating system has its own unique applications, formats, advantages and disadvantages, as discussed in several recent excellent critical reviews. ${ }^{1,3,5,7,16,18-}$ 20. Among the many available assessment tools, the Modified Barthel Index (MBI) and the Functional Independence Measure (FIM) are currently of special interest.

The Barthel Index, developed by Mahoney and Barthel ${ }^{13}$ and revised by Granger et al., ${ }^{14}$ has been used most frequently. When compared with other currently available assessment instruments, it has been considered a superior measure of functional ability because of its established reliability and validity, completeness, sensitivity to change in status, predictive value and clinical relevance. ${ }^{14,19,21-28}$ Despite several theoretical and practical problems, ${ }^{3,18}$ the $\mathrm{MBI}$ is considered the 'best buy' among all currently available scales. ${ }^{19,21,24,26-28}$ This scale has been used extensively in series of spinal cord injured (SCI) patients to document their functional capabilities, and to study differences in functional independence across various levels and extents of injury, ages and time intervals. ${ }^{29-31}$ These studies have demonstrated its internal consistency, reproducibility, ease of administration and utility when applied to serial assessments over time in this group.

The Functional Independence Measure (FIM) was developed to provide a uniform basis for the evaluation of function in patients with variety of disabilities. ${ }^{4}$ The USA regional model systems spinal cord injury care centres, supported by the National Institute on Disability and Rehabilitation Research of the USA Department of Education, has been conducting pilot studies utilizing the FIM as a basis for interval assessment of $\mathrm{SCI}$ patients. Preliminary reports ${ }^{32}$ indicate good reliability, validity, sensitivity and practicality of the FIM when applied to SCI patients.

The present investigation was designed to compare scores obtained on the MBI and on the FIM in SCI patients at serial assessment intervals.

\section{Methods}

\section{Subjects}

The study sample consisted of 41 acute traumatic (SCI) patients admitted within 45 days of injury to a regional model systems spinal cord injury care centre for acute management and comprehensive rehabilitation. All patients provided informed consent.

\section{Procedures}

Patient data were recorded, including age, gender, education, and level and aetiology of spinal cord injury. The abilities of each patient to perform functional activities were evaluated by one trained nurse-clinician using both the MBI and the FIM at three time periods: admission to initial rehabilitation (ADM), discharge from rehabilitation (DC) and at follow-up (FU) 12 months after rehabilitation discharge. Recording of functional scores was performed by only one evaluator in order to reduce the potential for unreliability of administration of the instrument.

The $\mathrm{MBI}$ is a 100 -point rating scale of a patient's ability to complete nine self-care (drinking, feeding, dressing upper body, dressing lower body, donning brace, grooming, bathing, bladder continence and bowel continence) and six mobility tasks (chair transfers, toilet transfers, tub transfers, walking 50 yards, stair climbing, and wheelchair propulsion), each of which is assigned a numeric value according to which of the three levels of assistance (independent, assistance, dependent) the patient's performance is rated at. ${ }^{13,14}$

The FIM is a similar rating system of a patient's 
Table 1 Items and scoring on the modified Barthel Index and the 'adapted' Functional Independence Measure

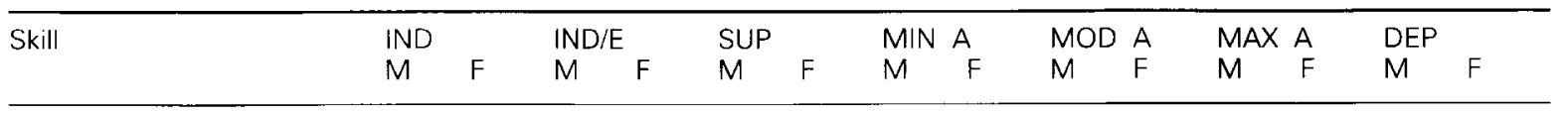

\section{Self-care subscore}

Drinking

Feeding

Upperdressing

Lower dressing

Grooming

Bathing

Bladder continence

Bowel continence

Perineal care

Mobility subscore

Chair transfers

Toilet transfers

Tub transfers

Walk on level

Stairs

Wheel

$\begin{array}{rrrr}4 & - & 4 & - \\ 6 & 7 & 6 & 6 \\ 5 & 7 & 5 & 6 \\ 7 & 7 & 7 & 6 \\ 5 & 7 & 5 & 6 \\ 6 & 7 & 6 & 6 \\ 10 & 7 & 10 & 6 \\ 10 & 7 & 10 & 6 \\ - & 7 & - & 6\end{array}$

$\begin{array}{ll}0 & - \\ 0 & 5 \\ 3 & 5 \\ 4 & 5 \\ 0 & 5 \\ 0 & 5 \\ 5 & 5 \\ 5 & 5 \\ - & 5\end{array}$

$\begin{array}{llllllll}0 & - & 0 & - & 0 & - & 0 & - \\ 0 & 4 & 0 & 3 & 0 & 2 & 0 & 1 \\ 3 & 4 & 3 & 3 & 0 & 2 & 0 & 1 \\ 4 & 4 & 4 & 3 & 0 & 2 & 0 & 1 \\ 0 & 4 & 0 & 3 & 0 & 2 & 0 & 1 \\ 0 & 4 & 0 & 3 & 0 & 2 & 0 & 1 \\ 5 & 4 & 5 & 3 & 0 & 2 & 0 & 1 \\ 5 & 4 & 5 & 3 & 0 & 2 & 0 & 1 \\ - & 4 & - & 3 & - & 2 & - & 1\end{array}$

IND = Independent

IND/E = Independent with equipment

SUP $=$ Independent with supervision

MINA = Independent with minimal assistance

MOD A $=$ Independent with moderate assistance

MAX $A=$ Independent with maximal assistance

DEP $=$ Dependent

$M=$ Modified Barthel Index ${ }^{14}$

$\mathrm{F}=$ Functional Independence Measure ${ }^{4}$

$\ldots=$ Not included on scale

ability to perform self-care, sphincter control, mobility, locomotion, communication, social adjustment and cognition tasks, each of which is rated on a scale between one and seven points, depending on the specific degree of assistance required for each task. ${ }^{4}$

In order to provide congruence between the two scales, an 'adapted' FIM score was used. The FIM subscales reflecting communication, social adjustment and cognition were deleted from analysis, and only the self-care (SC) and mobility (MO) subscales were studied in each of the two scales. It was previously demonstrated that the FIM subscales of communication and social cognition lacked external validity when compared to the results of a comprehensive neuropsychological battery. ${ }^{33}$ Subscale scores for self-care and sphincter control on the FIM were combined to create the FIM self-care subscore (which included feeding, grooming, bathing, dressing upper body, dressing lower body and perineal care tasks, while the subscores for mobility and locomotion on the FIM were summed to create the FIM mobility subscore (which included chair transfers, toilet transfers, tub transfers, walking, wheelchair propulsion and stair climbing).

Items on the MBI and the FIM, and their scoring systems, are listed in Table 1.

\section{Data Analysis}

Three 'static' scores (self-care subscore [SC], mobility subscore [MO] and total score [TO]) were generated for each of the two functional rating scales (the MBI and the FIM) at each of the three time periods (ADM, DC and FU) for each of the 41 patients. MBI scores and subscores were compared to FIM scores and subscores at ADM, DC and FU using simple linear regression analysis. Changes in each of the three scores and subscores $(\triangle \mathrm{SC}, \triangle \mathrm{MO}$ and $\triangle \mathrm{TO})$ were evaluated and compared between each of the two 
Table 2 FIM and MBI scores and subscores at admission, discharge, and follow-up (means \pm SEM)

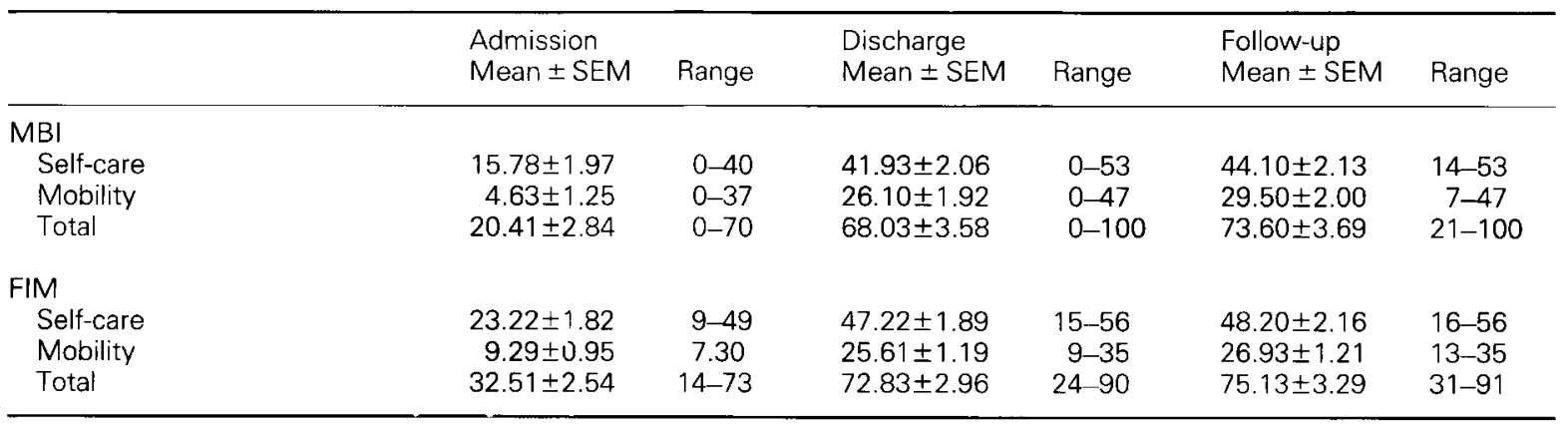

Table 3 Results of linear regression analysis of static MBI and FIM scores and subscores

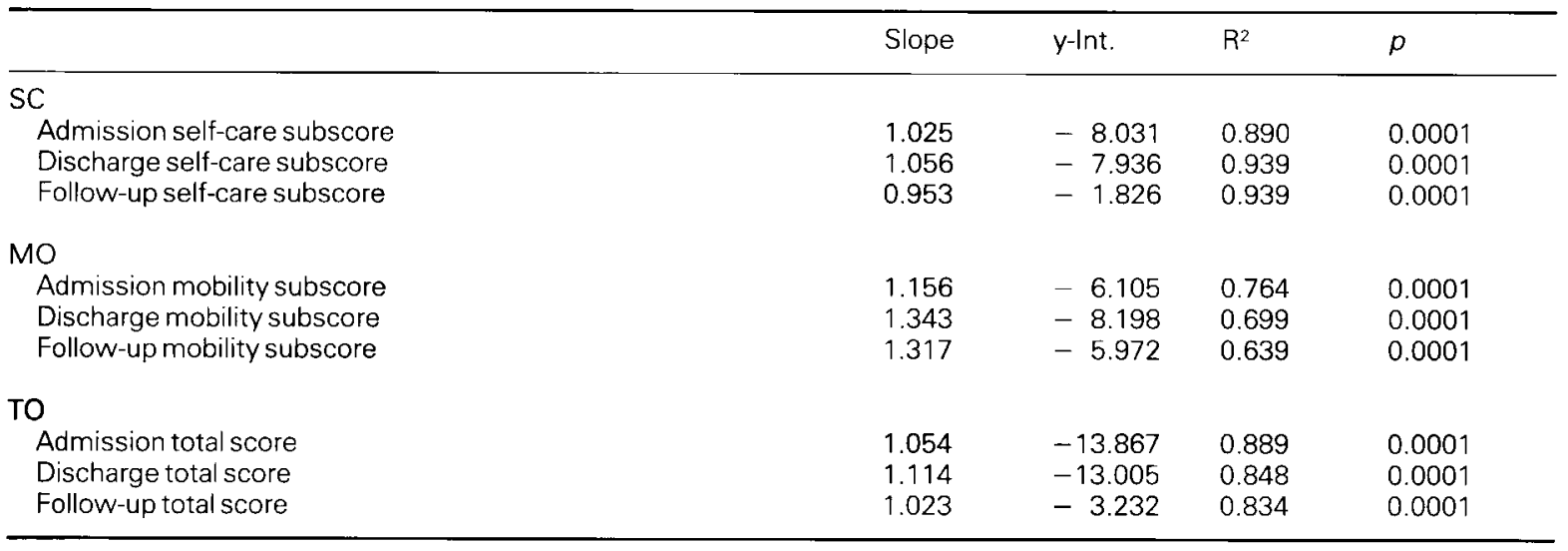

functional rating scales over each of the two time intervals (ADM-DC and DC-FU) for all patients using linear regression analysis.

\section{Results}

The mean $( \pm 1$ SEM) age of all patients was $30.4 \pm 1.7$ years. Thirty-five $(85 \%)$ of the patients were male. The mean educational level was $12.9 \pm 0.4$ years. Seventeen $(41 \%)$ patients sustained injuries at the cervical level, $16(39 \%)$ at the thoracic level and the remainder at the lumbosacral levels. Aetiologies of injury, in descending frequency, included: road traffic accidents, $29(70 \%)$; gunshot wounds, $4(10 \%)$; assaults, $4(10 \%)$; falls, $2(5 \%)$; and other causes, $2(5 \%)$.

Mean values and standard errors for MBI and FIM self-care and mobility subscores and total scores at admission, discharge and follow-up are shown in Table 2. There was a trend toward improvement in scores over time, especially between admission to and discharge from rehabilitation.

Each of the three static MBI and FIM scores and subscores at each of the three time periods (ADM, DC, FU) was compared using simple linear regression analysis (Table 3, Figures 1-3). This analysis revealed robust correlations between the MBI and the FIM for all parallel scores and subscores at all three time periods. Between $64 \%$ and $94 \%$ of the variance in the various FIM scores could be explained by $\mathrm{MBI}$ scores, with the most consistent correlations occurring for the three self-care subscore comparisons.

Changes in each of the three MBI scores and subscores $(\triangle \mathrm{MBI})$ and changes in each of the three FIM scores and subscores $(\triangle F I M)$ across 


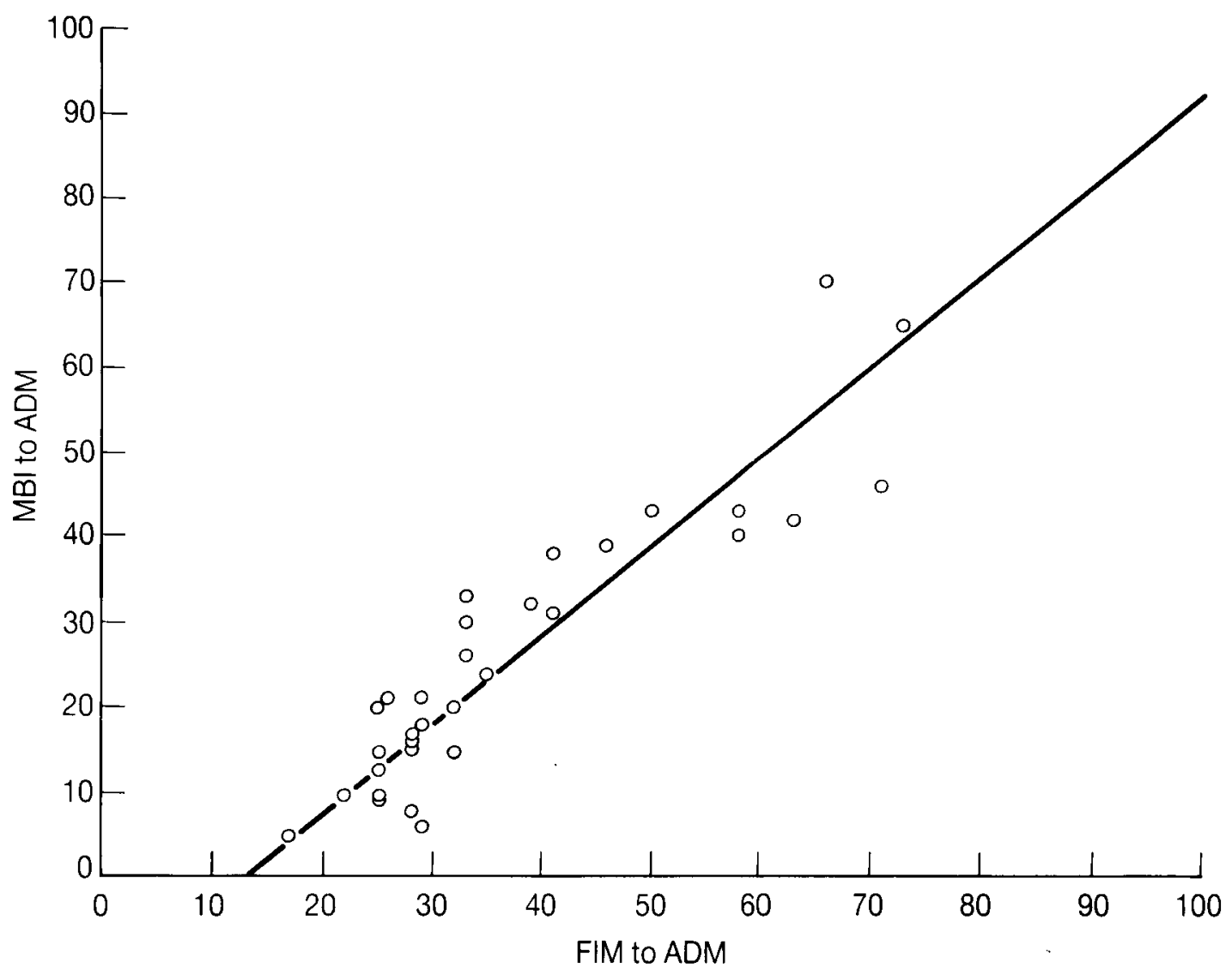

Figure 1 Regression analysis of FIM versus MBI scores: admission total scores $(y=1.054 x-13.867$.

R-squared $=0.889$ )

each of the two time intervals (ADM-DC and DC-FU) were also compared using simple linear regression analysis. This analysis demonstrated high degrees of correlation between changes in parallel MBI and FIM scores and subscores at all intervals studied. (Table 4). Between 36\% and $85 \%$ of the variance in FIM score changes could be explained by MBI score changes. Again, the highest degree of correlation was found in changes in the self-care subscores $(\triangle \mathrm{SC})$.

\section{Discussion}

Rating scales of ability to perform activities of daily living, such as the Modified Barthel Index (MBI) and the Functional Independence Measure
(FIM), have been developed to provide objective measures of functional status and change during and after acute and chronic rehabilitation of disabled individuals, including those with spinal cord injury. The Modified Barthel Index is considerably older, its validity more firmly established and its clinical usefulness more clearly demonstrated than the FIM. Because the FIM is a newer instrument, its role in the comprehensive assessment and management of SCI patients has not yet been fully elucidated, although several clinical evaluation trials of the FIM are currently underway. , $32^{2}$

Conceptually, the two scales are alike and are based on similar principles of evaluating the amount of assistance required for specific named daily living tasks. Indeed, the two systems assess 
Table 4 Results of linear regression analysis of changes in MBI and FIM scores over time $(\triangle M B I$ and $\triangle F I M)$

\begin{tabular}{|c|c|c|c|c|}
\hline & Slope & $y-\operatorname{lnt}$ & $R^{2}$ & $p$ \\
\hline \multicolumn{5}{|l|}{$\triangle \mathrm{SC}$} \\
\hline Admission-discharge self-care & 0.962 & 3.048 & 0.849 & 0.0001 \\
\hline Discharge-follow-up self-care & 0.847 & 0.774 & 0.748 & 0.0001 \\
\hline \multicolumn{5}{|l|}{$\triangle \mathrm{MO}$} \\
\hline Admission-discharge mobility & 1.063 & 4.222 & 0.542 & 0.0001 \\
\hline Discharge-follow-up mobility & 1.100 & 2.251 & 0.358 & 0.0005 \\
\hline \multicolumn{5}{|l|}{$\triangle \mathrm{TO}$} \\
\hline Admission-discharge total & 0.966 & 8.759 & 0.755 & 0.0001 \\
\hline Discharge-follow-up total & 0.856 & 3.628 & 0.645 & 0.0001 \\
\hline
\end{tabular}

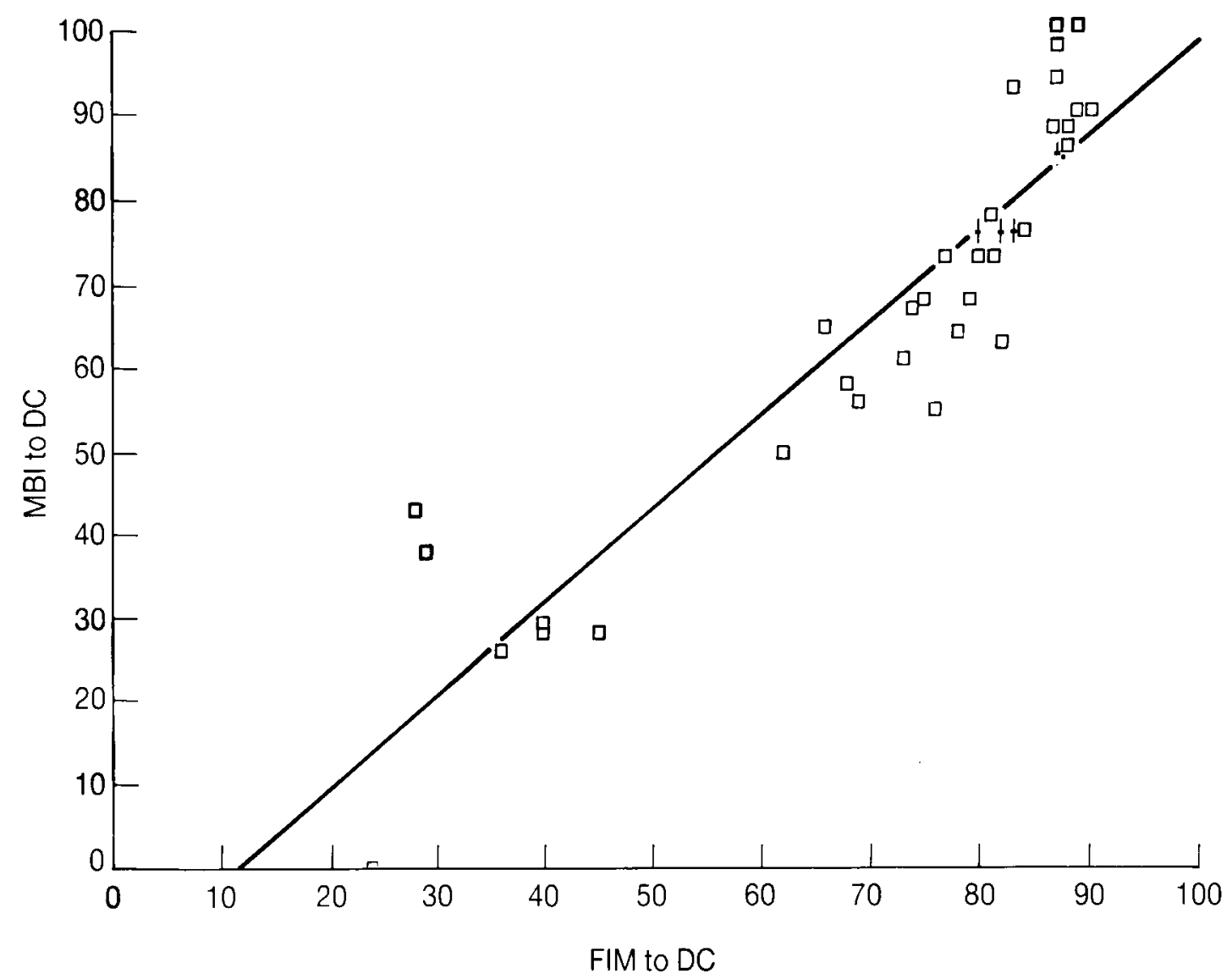

Figure 2 Regression analysis of FIM versus MBI scores: discharge total scores $(y=1.114 x-13.005$, R-squared $=0.848$ ) 


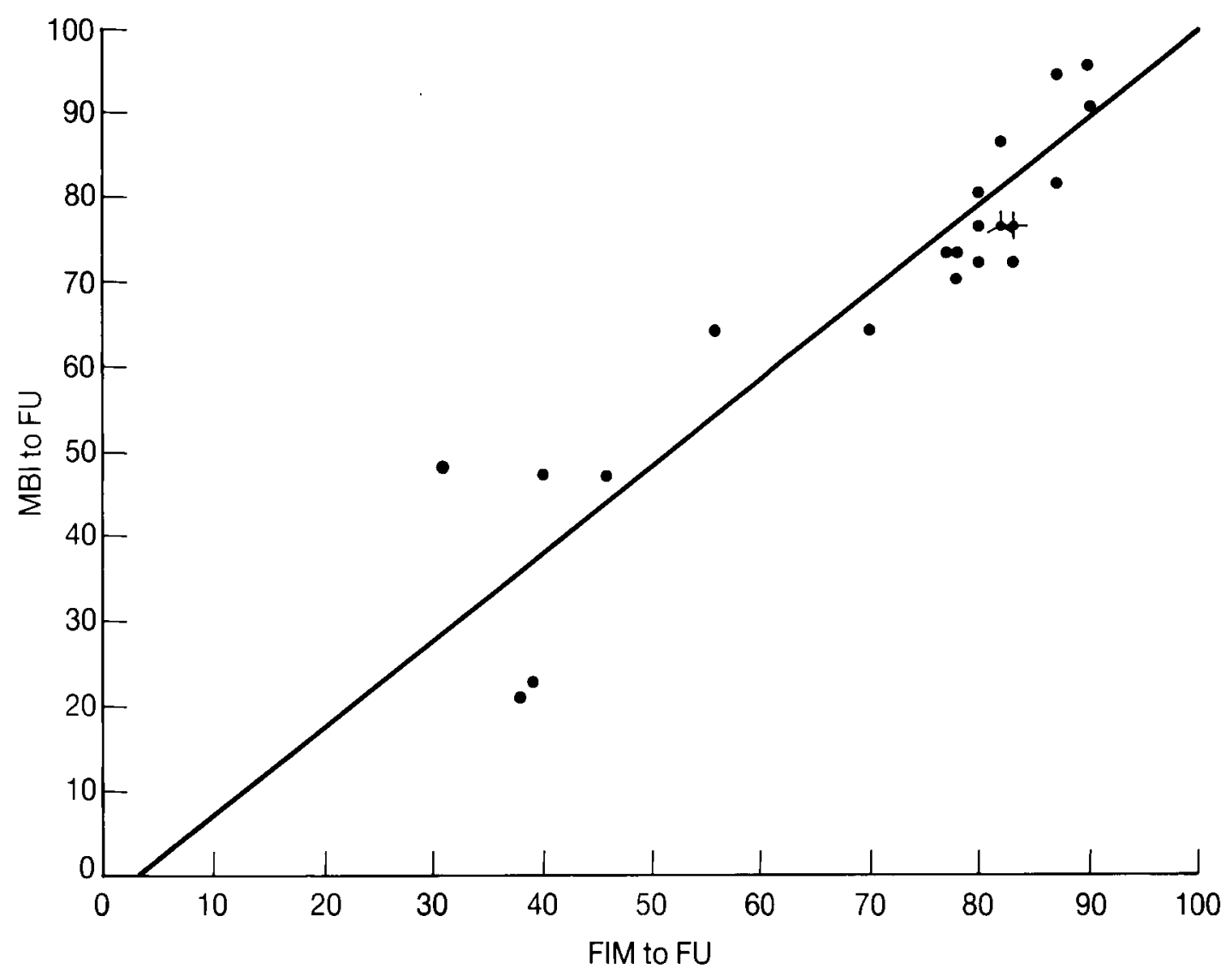

Figure 3 Regression analysis of FIM versus MBI scores: follow-up total scores $(y=1.023 x-3.232$, R-squared $=0.834$ )

nearly identical physical skills encompassing personal care and mobility aspects of functioning. It is therefore not completely surprising that scores on the two instruments were found to be as closely correlated as they were.

However, although these two assessment tools evaluate similar entities, they do so in different ways. At present both the rating and weighting systems of the two instruments are not alike. The FIM allows an individual to be rated on many more levels of assistance, which is especially important to fully evaluate those patients who require amounts of assistance or care in the 'middle' ranges. However, there is no indication on the FIM of the relative 'importance' of each task compared to the other skills. A recent report ${ }^{34}$ has suggested that the development and implementation of a weighting system for the FIM may be useful, but no such format exists as yet. On the other hand, the MBI has only three levels on which a patient may be rated for a specific task, thereby appearing to limit its sensitivity to small changes in functional status. However, there is a very clear and relatively intricate weighting system used for the MBI, by which the scores reflect the burden of care placed upon others as a result of the patient's particular level of functional disability.

Given these differences in the construction of these two rating systems, it is particularly striking that the analysis in this study yielded a high degree of correlation between the two scales. It is noteworthy that these robust statistical associations occurred for all scores and subscores, 
at all points in time (ADM, DC and FU) and across both time intervals (ADM-DC and DCFU).

A previous longitudinal study ${ }^{35}$ found that the Barthel Index and the FIM were very highly correlated with each other across several serial assessments for patients with stroke. The present investigation is the first to examine such a comparison for SCI patients.

The MBI and FIM scores and subscores for SCI patients were found to be highly correlated with each other across time. Because these two systems differ in their scoring format, but were found to yield scores which vary in a parallel fashion, selection of a specific rating system for a particular clinical or research application should be based on factors other than those studied in this investigation.

\section{Acknowledgements}

This paper was presented in part at the Fifteenth Annual Scientific Meeting of the American Spinal Injury Association, Las Vegas, on 4 April 1989.

This study was supported in part by a Clinical Investigator Development Award (GD) from the National Institute of Neurological Disorders and Stroke, National Institutes of Health, Bethesda, MD, USA (NS-01120-03) and by grants from the Kenny Michigan Rehabilitation Foundation, Southfield, MI, USA and the Spinal Cord Research Foundation of the Paralyzed Veterans of America, Washington DC, USA. Assistance in manuscript preparation was provided by Ms Jean Voeks.

\section{References}

1 Jette AM. State of the art in functional status assessment. In: Rothstein JM ed, Measurement in physical therapy, New York: Churchill Livingstone, 1985: 137-68.

2 Health and Public Policy Committee, American College of Physicians. Comprehensive functional assessment for elderly patients. Ann Intern Med 1988; 109: 70-72.

3 Feinstein AR, Josephy BR, Wells CK. Scientific and clinical problems in indexes of functional disability. Ann Intern Med 1986; 105: 413-20.
4 Granger CV, Hamilton BB, Keith RA, Zielezny M, Sherwin FS. Advances in functional assessment for medical rehabilitation. Top Geriatr Rehabil 1986; 1(3): 59-74.

5 Kane RA, Kane RL. Assessing the elderly: a practical guide to measurement. Lexington: Lexington Books, 1981.

6 Canadian Task Force on the Periodic Health Examination. The Periodic Health Examination. Can Med Assoc J 1979; 121: 1193-1254.

7 Granger CV, Gresham GE eds. Functional assessment in rehabilitation medicine. Baltimore: Williams \& Wilkins, 1984.

8 Rubenstein LZ, Josephson KR, Wieland GD, English PA, Sayre JA, Kane RL.

Effectiveness of a geriatric evaluation unit: a randomized clinical trial. N Engl J Med 1984; 311: $1664-70$.

9 Liem PH, Chernoff R, Carter WJ. Geriatric evaluation unit - A 3-year outcome evaluation. J Gerontol 1986; 41: 44-50.

10 Allen CM, Becker PM, McVay LJ et al. A randomized, controlled clinical trial of a geriatric consultation team: compliance with recommendations. JAMA 1986; 255: 2617-21.

11 National Institute on Aging Task Force. Senility reconsidered: treatment possibilities for mental impairment in the elderly. JAMA 1980; 244: 259-63.

12 Moskowitz E, McCann CB. Classification of disability in the chronically ill and aging. J Chron Dis 1957; 5: 342-46.

13 Mahoney F, Barthel D. Functional evaluation: the Barthel Index. Maryland State Med $J$ 1965; 14: 61-65.

14 Granger CV, Albrecht GL, Hamilton BB. Outcome of comprehensive medical rehabilitation: measurement by PULSES Profile and the Barthel Index. Arch Phys Med Rehabil 1979; 60: 145-54.

15 Schoening HA, Anderegg L, Bergstrom D, Fonda M, Steinke N, Ulrich P. Numerical scoring of self-care status of patients. Arch Phys Med Rehabil 1965; 46: 689-97.

16 Donaldson SW, Wagner CC, Gresham GE. A unified ADL evaluation form. Arch Phys Med Rehabil 1973; 54: 175-79.

17 Harvey RF, Jellinek HM. Functional performance assessment: a program 
approach. Arch Phys Med Rehabil 1981; 62: 456-61.

18 Keith RA. Functional assessment measures in medical rehabilitation: current status. Arch Phys Med Rehabil 1984; 65: 74-78.

19 Law M, Letts L. A critical review of scales of activities of daily living. Am J Occup Ther 1989; 43: 522-28.

20 Bruett TL, Overs RP. A critical review of 12 ADL scales. Phys Ther 1969; 49: 857-62.

21 Roy CW, Togneri J, Hay E, Pentland B. An inter-rater reliability study of the Barthel Index. Int J Rehab Research 1988; 11: 67-70.

22 Granger CV, Dewis LS, Peters NC, Sherwood $\mathrm{CC}$, Barrett JE. Stroke rehabilitation: analysis of repeated Barthel Index measures. Arch Phys Med Rehabil 1979; 60: 14-17.

23 Jacelon CS. The Barthel Index and other indices of functional ability. Rehabilitation Nursing 1986; 11: 9-11.

24 Gresham GE, Phillips TF, Labi MLC. ADL status in stroke: relative merits of three standard indexes. Arch Phys Med Rehabil 1980; 61: 355-58.

25 Jongbloed L. Prediction of function after stroke: a critical review. Stroke 1986; 17: 76576.

26 Collin C, Wade DT, Davies S, Horne V. The Barthel ADL Index: a reliability study. Int Disabil Stud 1988; 10: 61-63.

27 Wade DT, Langton-Hewer R. Functional abilities after stroke: measurement, natural history and prognosis. I Neurol Neurosurg Psychiatry 1987; 50: 177-82.
28 Wade DT, Collin C. The Barthel ADL Index: a standard measure of physical disability? Int Disabil Stud 1988; 10: 64-67.

29 Yarkony GM, Roth EJ, Heinemann AW, Wu YC, Katz RT, Lovell L. Benefits of rehabilitation for traumatic spinal cord injury: multivariate analysis in 711 patients. Arch Neurol 1987; 44: 93-96.

30 Yarkony GM, Roth EJ, Heinemann AW, Lovell LL. Spinal cord injury rehabilitation outcome: the impact of age. J Clin Epidemiol 1988; 41: 173-77.

31 Yarkony GM, Roth EJ, Heinemann AW, Lovell L, Wu YC. Functional skills after spinal cord injury rehabilitation: three-year longitudinal follow-up. Arch Phys Med Rehabil 1988; 69: 111-14.

32 Whiteneck GG. A Functional Independence Measure trial in SCI model systems. Proc Am Spinal Injury Assoc 1988; 14: 48.

33 Davidoff G, Roth E, Haughton J, Ardner M. Cognitive dysfunction in spinal cord injury patients: sensitivity of the Functional Independence Measure subscales versus neuropsychological assessment. Arch Phys Med Rehabil 1989, in press.

34 Dijkers MP, Development of item weights for the Functional Independence Measure: pilot study. Arch Phys Med Rehabil 1988; 69: 755.

35 Wagner MT, Zucchigna LJ. Longitudinal comparison of the Barthel and FIM during the first six months of recovery from stroke. Arch Phys Med Rehabil 1988; 69: 755. 